Kardiologe $2016 \cdot 10: 47-55$

DOI 10.1007/s12181-015-0035-3

Online publiziert: 14. Januar 2016

(c) Die Autoren 2015. Dieser Artikel ist auf

SpringerLink mit Open Access verfügbar

CrossMark

H. von Bibra' · W. Paulus ${ }^{2}$

'Klinik für Endokrinologie, Diabetologie und Angiologie, Städt. Klinikum Bogenhausen, München, Deutschland

${ }^{2}$ Institute for Cardiovascular Research Vrije Universiteit, VU University Medical Center Amsterdam, Amsterdam, Niederlande

\title{
Diastolische Dysfunktion
}

\section{Echokardiographische Erfassung, Ätiologie und Therapieansätze}

erklärt auch den mangelnden Konsens zu Definition und echokardiographischer Diagnostik [13, 22, 35, 37]. Dieser Beitrag stellt weiterführende aktuelle und klinisch relevante Forschungsergebnisse vor, die v. a. frühe und reversible pathophysiologische Mechanismen im Fokus haben und mittels mathematisch korrekterer echokardiographischer Diagnostik identifiziert und als Grundlage für präventive oder therapeutische Ansätze dienen können.

\section{Definition und echokardiogra- phische Bestimmungskriterien}

In der Abfolge des kardialen Zyklus setzt sich die LV diastolische Funktion zusammen aus Relaxation sowie frühdiastolischer Füllung als aktive und energieverbrauchende myokardiale Dehnung und der spätdiastolischen Füllung durch Vorhofkontraktion. DD, d. h. Störungen dieser Abläufe, sind Grundlage von HFpEF und in den vorrausgehenden subklinischen Phasen mit insbesondere frühdiastolisch gestörter Funktion sensible Indikatoren einer myokardialen Schädigung, häufig auf reversibler Grundlage und insofern klinisch relevant für Diagnostik und präventive Therapie.

Die nichtinvasiv bestimmte DD ist in den europäischen Leitlinien zur Diagnostik von diastolischer Herzinsuffizienz [22] definiert als eine auf Gewebedoppler basierende Abschätzung des LV Füllungsdruckes $\mathrm{E} / \mathrm{E}^{\prime}>15 \mathrm{bzw}$. für den Bereich $15<$ E/E' $<8$ (• Abb. 1) mit komplexen Algorithmen unter Einschluss von echokardiographischen strukturellen $\mathrm{Pa}$ - rametern von LA und LV sowie Biomarkern. Diese multiplen nichtinvasiven Kriterien haben sich in der praktischen Routine für die Diagnostik von HFpEF als teilweise nicht sensitiv oder unschlüssig erwiesen [13, 19, 24], fraglich ist auch eine Eignung zur Früherkennung der DD für die effektive Prävention einer Herzinsuffizienz. Die Limitationen von E/E' und strukturellen LV bzw. LA Parametern als diagnostische Kriterien der DD bedingen einen wachsenden Bedarf an sensitiver Quantifizierung der DD auf einer Grundlage, die der Dynamik der Veränderungen der LV diastolischen Funktion Rechnung trägt $[8,35]$.

Die traditionell in der Praxis üblichen echokardiographischen Methoden der diastolischen Funktionsbestimmung über den Mitralklappeneinstrom (E/A) sind inzwischen durch quantifizierende Funktionsparameter mittels Gewebedoppler (frühdiastolische Myokardgeschwindigkeit E' und spätdiastolische A') ergänzt bzw. ersetzt worden (• Abb. 2), zumal der LV Füllungsdruck hiermit als E/E' abgeschätzt werden kann [22].

\begin{tabular}{|ll}
\hline \multicolumn{2}{|l}{ Abkürzungen } \\
\hline$B M I$ & Body-Mass-Index \\
\hline$D D$ & Diastolische Dysfunktion \\
\hline$E F$ & Ejektionsfraktion \\
\hline$H F p E F$ & $\begin{array}{l}\text { Heart failure preserved ejection } \\
\text { fraction }\end{array}$ \\
\hline$L A$ & Linksatrial \\
\hline$L V$ & Linksventrikulär \\
\hline
\end{tabular}

Verständnis der pathophysiologischen Mechanismen der DD [9, 25, 28, 32, 37] 


\section{Evidenz für}

abnorme LV-Relaxation, Füllung, diastolische Dehnbarkeit und Steifigkeit

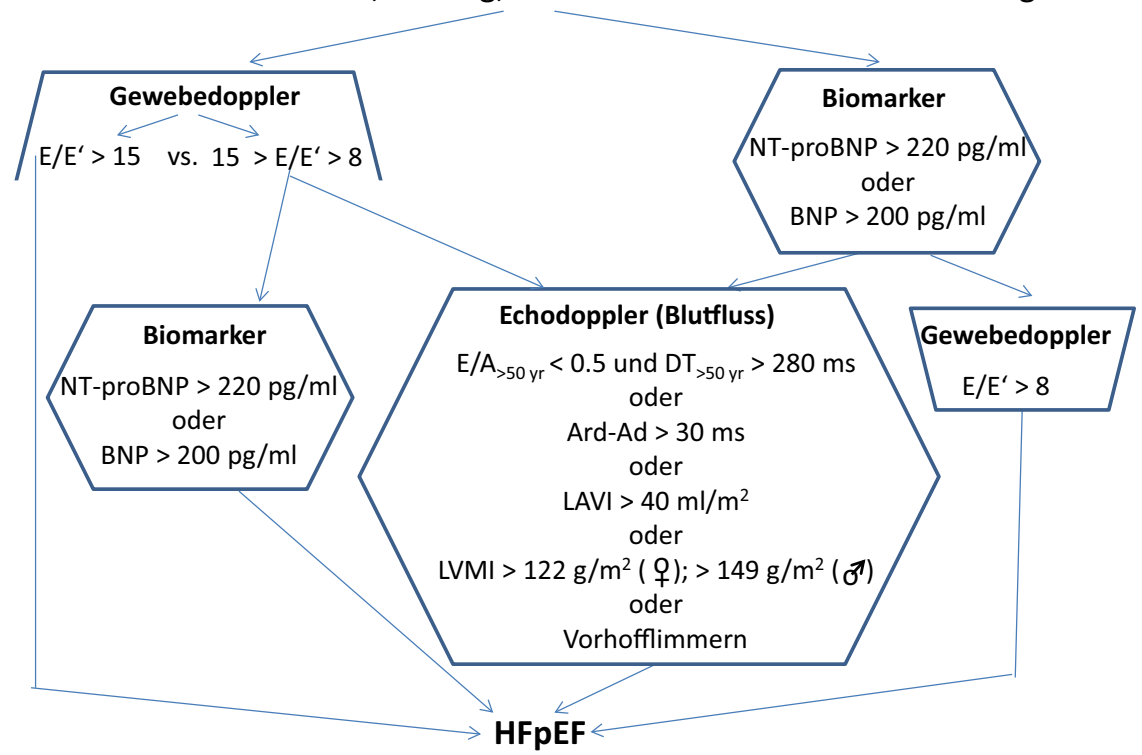

Abb. $1 \Delta$ Leitliniengerechte Definitionen zu DD in der nichtinvasiven Diagnostik von HFpEF. E frühdiastolische mitrale Flussgeschwindigkeit, $E^{\prime}$ frühdiastolische Myokardgeschwindigkeit, NT-proB$N P{ }_{\text {"N}} \mathrm{N}$-terminal-pro brain" natriuretisches Peptid, $B N P$ "brain natriuretic" Peptid, E/A Quotient früh(E) zu spätdiastolischer (A) mitraler Flussgeschwindigkeit, DT Dezelerationszeit, LVMI LV Massenindex; LAVI LA Volumenindex, Ard Dauer des retrograden pulmonalvenösen systolischen LAFlusses, Ad Dauer des mitralen spätdiastolischen Flusses. (Modifiziert nach [22])

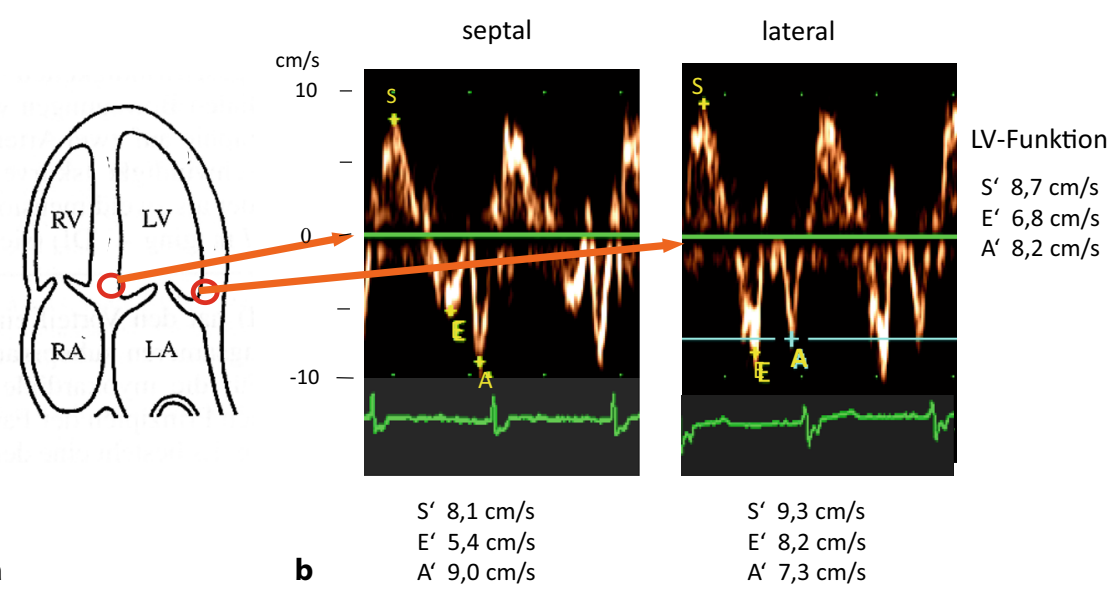

Abb. 2 ॥ Bestimmung der globalen LV Funktion mit gepulstem Gewebedoppler als Mittelwert von 2 bis 6 Messungen in basalen Myokardabschnitten, exemplarisch anhand a der 4-Kammer-Schau und b der dabei erzielten Geschwindigkeitszeitkurven septal und lateral. $S^{\prime}$ systolische Geschwindigkeit, $E^{\prime}$ frühdiastolische Geschwindigkeit, $A^{\prime}$ spätdiastolische Geschwindigkeit

Allerdings konnte bisher kein Funktionskriterium als zuverlässiges $\mathrm{Maß}$ der aktiven Myokardrelaxation etabliert werden, obgleich Gewebedopplermessungen mit E, einem sensitiven Parameter frühdiastolischer Myokardfunktion mit gutem prognostischem Aussagewert, seit Langem zur Verfügung stehen $[17,21$, 40]. Inzwischen wurde auch der positive
Zusammenhang zwischen therapeutischen Veränderungen von $E^{\prime}$ und der klinischen Leistungsfähigkeit aufgezeigt [7, 12]. Als limitierender Faktor erwies sich die ausgeprägte Altersabhängigkeit von E' [35], wobei die physiologische altersbedingte Abnahme der frühdiastolischen Funktion seit Langem bekannt ist [37]. Bei gesunden Individuen verrin- gert sich E' jährlich um $1 \%$ (• Abb. 3), d. h. von $17 \mathrm{~cm} / \mathrm{s}$ im 20. Lebensjahr auf $12 \mathrm{~cm} / \mathrm{s}$ mit 50 Jahren und auf $7 \mathrm{~cm} / \mathrm{s}$ mit 80 Jahren, also insgesamt um $59 \%$. Es ist jedoch gerade die enge und steile Korrelation von E' und Alter, die eine Quantifizierung der DD ermöglicht. Wie dominant das Alter die diastolische Funktion beeinflusst, ist erst kürzlich mittels Regressionsanalyse durch den Regressionskoeffizienten $\beta$ mit 0,88 ermittelt worden, d.h., dass die Prognose von $\mathrm{E}^{\prime} \mathrm{zu} 88 \%$ von der unabhängigen Variablen Alter bestimmt wird [8]. Daraus folgt, dass die normale diastolische Funktion über die Altersabhängigkeit definiert werden muss: Die entsprechende lineare Regressionsgleichung von $\mathrm{E}_{\text {norm }}$ anhand gesunder Individuen erlaubt die diagnostische Zuordnung im Vergleich zur individuell gemessenen E' (• Abb. 3 und 4). DD ist somit unterhalb des $95 \%$ Konfidenzintervalls (• Abb. 3 ) definiert $\left(E^{\prime}-E_{\text {norm }}^{\prime}>2,8 \mathrm{~cm} / \mathrm{s}\right)$ und ermöglicht nun die korrekte Identifizierung von determinierenden Risikofaktoren in entsprechenden Regressionsgleichungen. Stattdessen waren bisher in den Korrelationen zu E' v. a. diejenigen Risikofaktoren signifikant erschienen, die ihrerseits stark altersabhängig sind. Neue Analysen der DD bei 205 nichtdiabetischen Individuen haben folgende Risikofaktoren als Determinanten ergeben: vaskuläre Steifigkeit, gemessen als Pulswellengeschwindigkeit, diastolischer Blutdruck und Insulinresistenz, abgeschätzt als Quotient Serumtriglyzeride/ High-density-Lipoprotein (HDL), während für die 204 diabetischen Patienten dieselben Risikofaktoren in umgekehrter Reihenfolge bestätigt wurden. LV und LA strukturelle Parameter erreichten keine Signifikanz [8].

Im Gegensatz zur frühdiastolischen Funktion muss die normale systolische Funktion S' nicht über das Alter definiert werden, da sie, nur zu $39 \%$ vom Alter bestimmt, jährlich nur um 0,3\% abnimmt [8].

\section{Prävalenz und Klinik}

Die Häufigkeit von DD in der Bevölkerung wurde mit 11-27\% [36] angegeben und für subklinische DD mit 20-30 \% [1, 
26]. Prognostisch hat DD große Bedeutung für eingeschränkte Lebensqualität und erhöhtes kardiovaskuläres Risiko, für die Weiterentwicklung zu Herzinsuffizienz und für Mortalität [1, 21, 26].

Klinisch sind Belastungsdyspnoe und/ oder Leistungsabfall führend. Unauffällige diastolische Funktionsmessungen in Ruhe sollten durch Belastungsuntersuchungen (zumindest 6-min-Gehtest) ergänzt werden, denn eingeschränkte diastolische Reserve führt evtl. auch zu lebensqualitätsminderndem Leistungsabfall. Trotz der bekannten Risikofaktoren Übergewicht, metabolisches Syndrom und Diabetes wird DD bei diesen Patienten selten diagnostiziert: Einerseits verschweigen die resignierten und „verschämten Dicken“ ihre Leistungseinschränkung häufig, andererseits sind die traditionellen Untersuchungsmethoden EKG, Thoraxröntgenaufnahme und Brain Natriuretic Peptide unspezifisch [4, 21].

\section{Diagnostik}

In der Praxis hat sich der gepulste Gewebedoppler als sensitive, robuste, allgemein verfügbare und auch bei schwer schallbaren Patienten praktikable Methodik bewährt [5, 10, 13, 37]. Wichtig sind dabei für reproduzierbare Messungen von E' (• Abb. 5)

- die apikale Ableitung in der unverkürzten LV Längsachse, d. h. im tiefst möglichen Interkostalraum,

- die Minimierung von Winkelfehlern durch Zentrierung des LV Apex im echokardiographischen Sektor,

- die Bestimmung der globalen LV Funktion durch Mittelung von E' aus Messungen in beiden Wänden des 4-Kammer-Blicks, besser auch des 2Kammer-Blicks,

- bei Farbdopplerverfahren: eine hohe Bildaufbaugeschwindigkeit $>100 / \mathrm{s}$ [4].

Für gepulsten Gewebedoppler lautet die lineare Regressionsgleichung zur Errechnung von $E_{\text {norm }}^{\prime}$ [8] bei:

- 2 Messungen (4-Kammer-Blick;

- Abb. 2): $E_{\text {norm }}^{\prime}=-0,15 \times$ Alter

(Jahren) $+18,9(\mathrm{~cm} / \mathrm{s})$,

Kardiologe 2016 $10: 47-55 \quad$ DOI 10.1007/s12181-015-0035-3

(c) Die Autoren 2015

H. von Bibra · W. Paulus

\section{Diastolische Dysfunktion. Echokardiographische Erfassung, Ätiologie und Therapieansätze}

\section{Zusammenfassung}

Mit veränderter Altersstruktur, Diabetesund Adipositasprävalenz der Bevölkerung nimmt auch die Prävalenz von Herzinsuffizienz epidemisch zu. Dabei leidet ca. die Hälfte aller Betroffenen überwiegend an diastolischer Dysfunktion (DD) bei erhaltener LV Ejektionsfraktion (HFpEF) und hat damit eine ebenso ungünstige Prognose wie bei systolischer Herzinsuffizienz, bislang jedoch keine evidenzbasierten Therapieoptionen. Derzeit ist das Verständnis der zugrunde liegenden pathophysiologischen Mechanismen unvollständig und zusätzlich besteht ein mangelnder Konsens bezüglich Definition und Diagnostik der DD. Zum Problem einer unzureichenden myokardialen Energieversorgung tragen die bekannten ätiologischen Faktoren Alter, Hypertonus, koronare Herzerkrankung, Diabetes, Insulinresistenz, Adipositas, Anämie, chronisch obstruktive Lungenerkrankung, Schlafapnoe und Niereninsuffizienz ursächlich bei. Insbesondere muss das Lebensalter als wichtigste und dominierende Determinante der diastolischen Funktion beachtet werden. Zur Quantifizierung der DD benötigt man die individuell mit Gewebedoppler gemessene diastolische Myokardfunktion E' und zur vergleichenden Korrektur die Regressionsgleichung von $\mathrm{E}_{\text {'norm }}$ als Funktion des Alters von gesunden Individuen. Die Differenz $E^{\prime}-E_{\text {norm }}^{\prime}$ ermöglicht die Definition von DD und gleichzeitig auch die Evaluation altersunabhängiger Veränderungen aus strukturellen, hämodynamischen und auch metabolischen Einflüssen. Eine wesentliche Bedeutung kommt dem metabolischen Risiko, insbesondere der Insulinresistenz, für die reduzierte Energieversorgung bei DD zu. Durch die Reversibilität der Insulinresistenz ergibt sich die Option einer fokussierten Therapie, die durch geeignete Studien belegt werden muss.

Schlüsselwörter

Diastolische Funktion · Alter · Herzinsuffizienz · Insulinresistenz · Kardiovaskuläre Risikofaktoren

\section{Diastolicdysfunction. Echocardiographic detection, etiology and therapy}

\begin{abstract}
Heart failure is an ongoing epidemic of growing dimensions in western civilizations due to the increasing prevalence of the predisposing risk factors age, diabetes and obesity. Approximately one half of the patients have predominantly diastolic dysfunction (DD) and preserved left ventricular ejection fraction (HFpEF) with a prognosis that is just as unfavorable as systolic heart failure but with no known effective treatment. This stems from several factors, such as incomplete understanding of the underlying pathophysiological mechanisms and a lack of consensus on how to define and diagnose DD. All the known risk factors for DD and HFpEF such as age, hypertension, coronary artery disease, diabetes, insulin resistance, obesity, anemia, chronic obstructive pulmonary disease, sleep apnea and renal insufficiency are associated with insufficient availability
\end{abstract}

of myocardial energy. The dominant impact of age on diastolic function $\mathrm{E}^{\prime}$, as measured by tissue Doppler, requires as mathematical solution the comparison of the individually measured $\mathrm{E}^{\prime}$ to a regression equation of $E_{\text {norm }}$ over age from healthy individuals. This reference data allows the definition and the quantification of DD and the evaluation of age-independent structural, hemodynamic and metabolic risk factors for DD. This approach reveals the importance of metabolic risk and provides new options for therapy and for studies with well-defined inclusion criteria via the potential reversibility of insulin resistance.

Keywords

Diastolic function · Age $\cdot$ Heart failure $\cdot$ Insulin resistance $\cdot$ Cardiovascular risk factors 
$(\mathrm{cm} / \mathrm{s})$

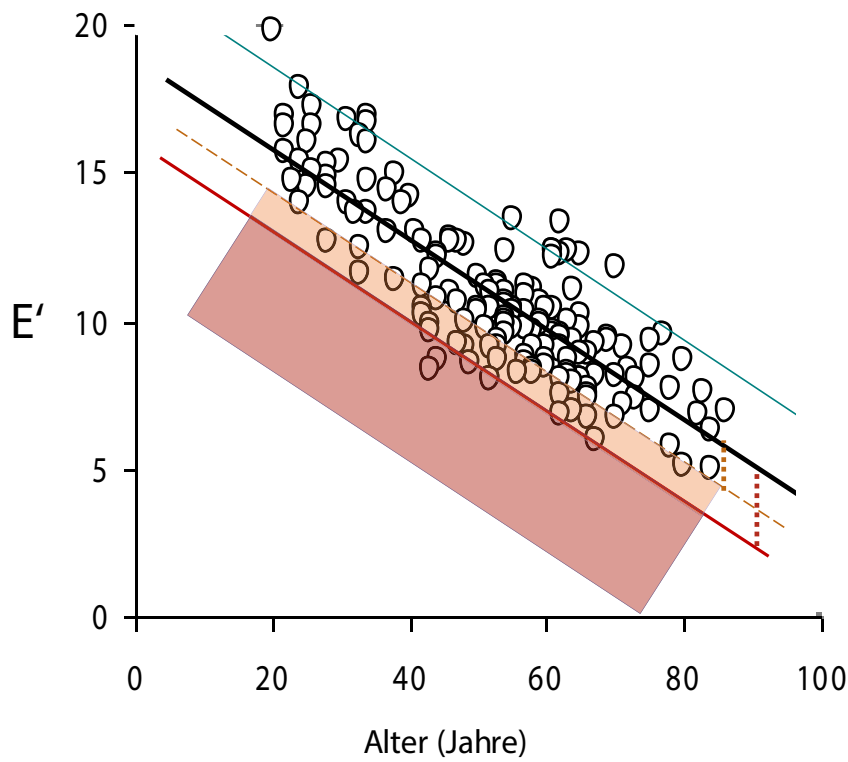

Abb. $3 \Delta$ E' als Funktion des Alters bei 205 nichtdiabetischen Kontrollen mit der Regressionslinie (-) E'norm $=-0,158 \times$ Alter $+19,6$, sowie der oberen (blau) und unteren (rot) $95 \%$-Konfidenzlinie, unterhalb derer DD definiert ist (rötliches Feld) und angrenzend Risiko für DD (blaßoranges Feld). (Modifiziert nach [8]) $(\mathrm{cm} / \mathrm{s})$

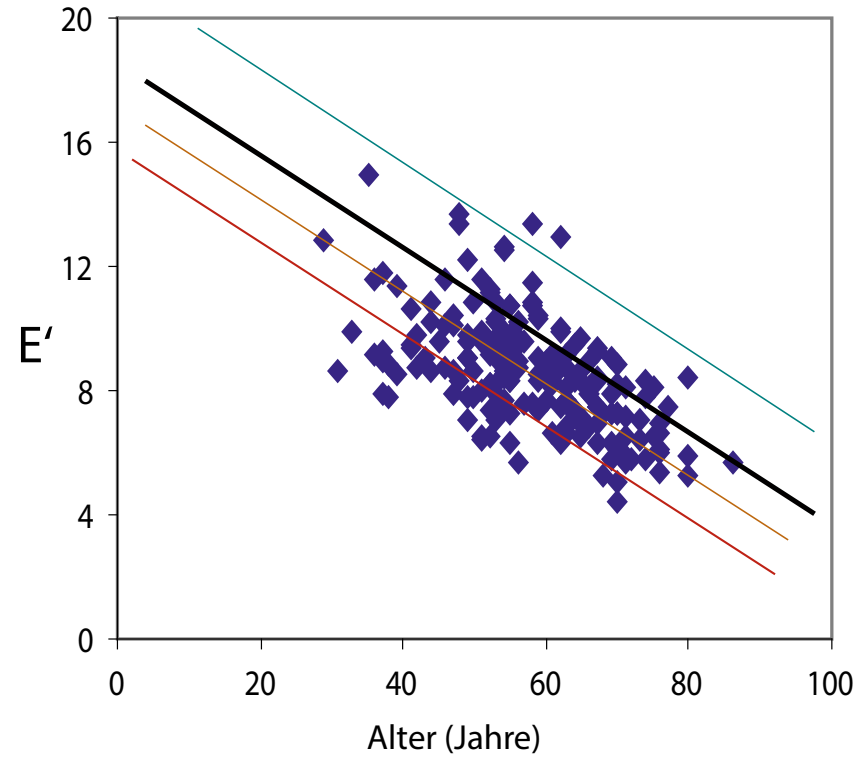

Abb. $4 \Delta$ E' als Funktion des Alters bei 204 Diabetikern mit identischen Regressionsgraden und $95 \%$-Konfidenzlinien wie $\bullet$ Abb. 3 zeigt für Typ 2 Diabetes mit $34 \%$ eine hohe Prävalenz von DD (unterhalb der unteren Konfidenzlinie) verglichen mit Risiko fürDD (37\%) und normaler Funktion (28\%). (Modifiziert nach [8])

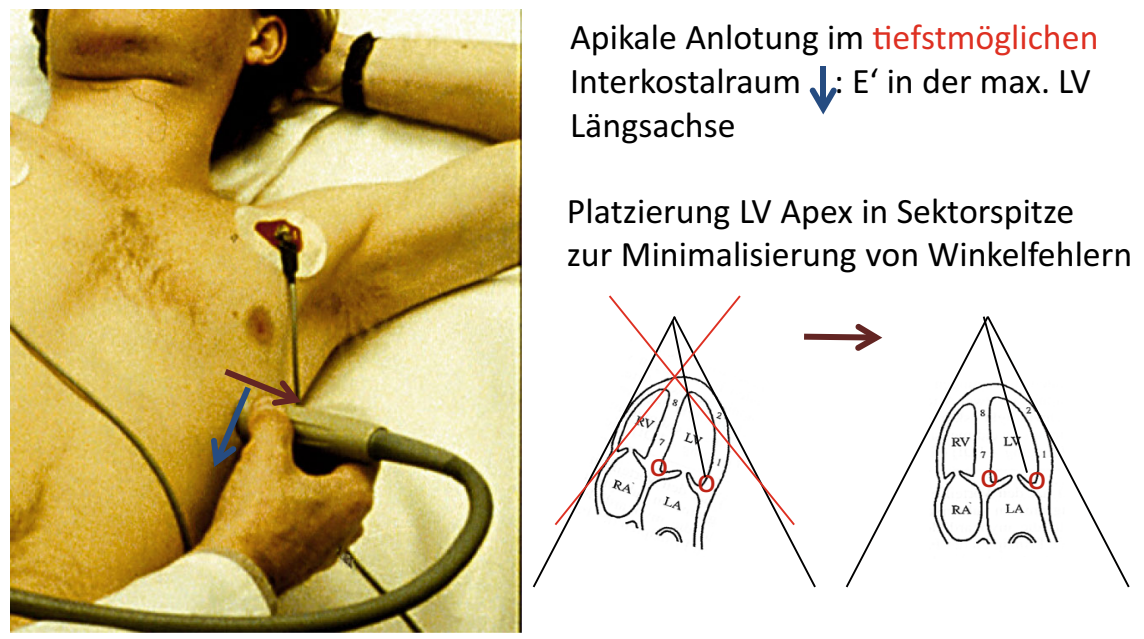

Abb. 5 ॥ Bei der Messung mit Gewebedoppler Optimierung der Transducerposition für die Ableitung in der maximalen LV Längsachse $\downarrow$ und für die Minimierung von Winkelfehlern $\rightarrow$

- 4 Messungen (4- und 2-KammerBlick): $\mathrm{E}_{\text {norm }}^{\prime}=-0,158 \times$ Alter (Jahren) $+19,6(\mathrm{~cm} / \mathrm{s})$

- 6 Messungen (2-, 3- und 4-Kammer-Blick): $\mathrm{E}_{\text {norm }}=-0,163 \times$ Alter (Jahren) $+19,6(\mathrm{~cm} / \mathrm{s})$.

Wie - Abb. 3 erläutert, ist DD definiert als E' unterhalb der unteren $95 \%$-Konfidenzlinie der Regressionsgeraden des Normalkollektivs E' ${ }_{\text {norm. }}$. Praktisch wird vom Messwert E' das errechnete E' abgezogen. Ist das Defizit zu $\mathrm{E}_{\text {norm }}$ gröBer als $2,8 \mathrm{~cm} / \mathrm{s}$, besteht eine DD, ist es zwischen 1,4 und $2,8 \mathrm{~cm} / \mathrm{s}$ besteht ein Risiko für DD und bei kleinerem Defizit eine normale Funktion [8].

Grundsätzlich gilt dieses Prinzip völlig analog auch für Messungen der diastolischen Funktion mittels Gewebe-Farbdoppler-Echokardiographie und andere Imaging Techniken mit aus- reichender Bildaufbaurate (> 100/s). Die entsprechenden Regressionsgleichungen und Konfidenzintervalle von Normalkollektiven müssen jedoch noch bestimmt werden. Messungen mittels Magnetresonanztechnik sind durch die limitierte Zeitauflösung/Bildaufbaurate ungeeignet $[4,37]$.

Für die Diagnostik einer DD ist die Vorhofvergrößerung (LA Volumenindex $>28 \mathrm{ml} / \mathrm{m}^{2}$ ) ein sensitiver, aber unspezifischer Zusatzparameter [8, 22], da eine Elongation des linken Vorhofs die erste Reaktion auf eine Volumenbelastung durch gestörte LV Relaxation jeglicher Ätiologie ist [16, 33, 35]. Bei unzureichender Schallqualität für die LA Volumenbestimmung sollte die Vergrößerung des linken Vorhofs als Fläche im 4-Kammer-Blick (LA Flächenin$\operatorname{dex}>28 \mathrm{~cm}^{2} / \mathrm{m}^{2}$ bzw. LA Fläche $>20 \mathrm{~cm}^{2}$ für durchschnittlich große/schwere Patienten; [20]) oder zumindest dessen Längsdurchmesser neben dem interatrialen Septum bestimmt werden (LA Diameterindex $>2,8 \mathrm{~cm} / \mathrm{m}^{2}$ bzw. LA Diameter $>5 \mathrm{~cm}$ für durchschnittlich große/schwere Patienten), da der traditionell bestimmte anterior-posteriore Durchmesser für LA Elongation unsen- 


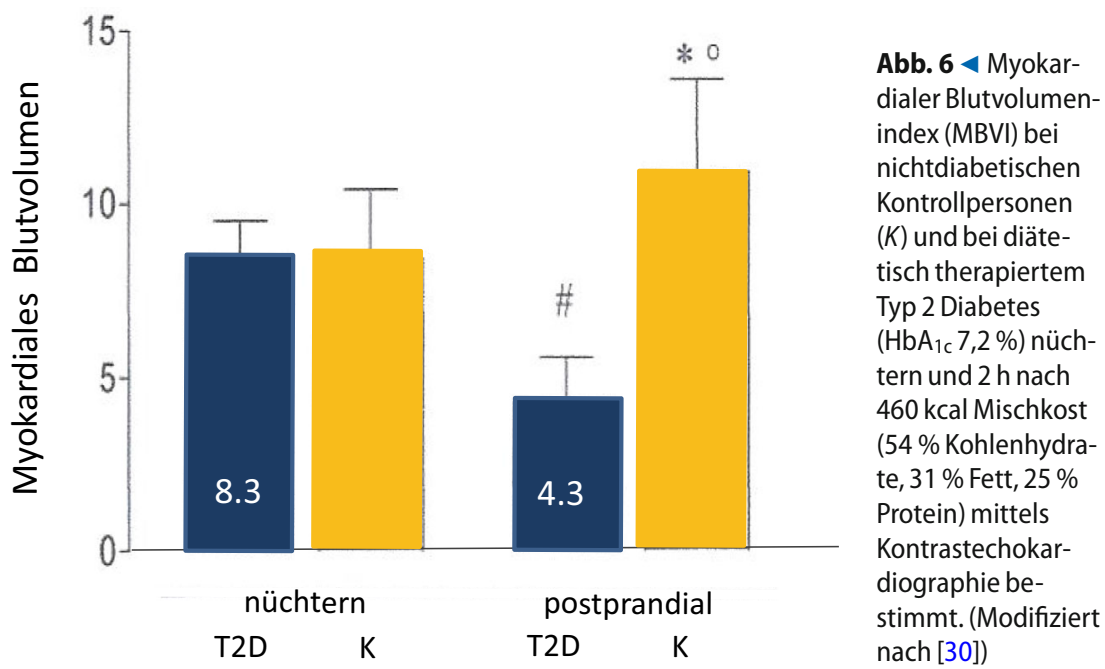

sitiv und damit weniger prognostisch ist [34].

\section{Ätiologie/pathophysiologische Mechanismen}

In dieser Übersicht sollen die klinisch dominanten, kardiovaskulären und systemischen Grundlagen von DD genannt werden. Seltenere Entitäten wie hypertrophische Kardiomyopathie oder myokardiale Speicherkrankheiten werden nicht berücksichtigt.

Von grundlegender Bedeutung ist die aus der Ischämiekaskade resultierende Erkenntnis, dass unzureichende myokardiale Sauerstoff-/Energieversorgung zuerst zur Reduktion der extrem energieabhängigen frühdiastolischen und erst verzögert der systolischen Funktion führt [32]. Diese Auswirkung erfolgt sofort, ist reversibel und ohne strukturelle Veränderungen. Eine intakte frühdiastolische Funktion trägt zu ca. 80 \% des Schlagvolumens bei. Mitzunehmendem Lebensalter muss bei abnehmender frühdiastolischer Myokardfunktion mehr Vorhofarbeit gegen potenziell zunehmende myokardiale Steifigkeit im linken Ventrikel geleistet werden.

Als Grundlage klinisch therapeutischer Überlegungen bei DD ergibt sich ein vereinfachtes 2 -Stufen-Konzept. Infolge der bekannten und häufigsten ätiologischen Faktoren/Komorbiditäten Alter, Hypertonus, koronare Herzerkrankung, Diabetes, Adipositas, chronisch obstruktive Lungenerkrankung,
Diastolische Dysfunktion und Adipositas/Insulinresistenz

Die Diagnose „obesity cardiomyopathy“ [39] macht den Zusammenhang von Adipositas und DD deutlich. Hohe Insulinspiegel und Insulinresistenz bewirken durch direkte metabolische Einflüsse eine bis zu 40 \%ige Reduktion in der Effizienz kardiozytärer AdenosintriphosphatProduktion $[9,38]$. Zusätzliche indirekte Effekte bestehen durch Insulinresistenz, oxidativen Stress, verminderte Stickstoffbioverfügbarkeit sowie Lipotoxizität, die vermittels chronisch inflammatorischer Prozesse und endothelialer Dysfunktion zur Fehlregulierung der myokardialen Perfusion führen $[5,23]$. Es resultiert ein myokardialer Energie-Mismatch angesichts des erhöhten Sauerstoffbedarfs für das adipositasbedingte erhöhte Schlagvolumen und führt zur Reduktion der besonders energieintensiven frühdiastolischen Myokardfunktion.

\section{Diastolische Dysfunktion und Diabetes}

Hyperglykämie kann direkt postprandial über oxidativen Stress zur kardialen Funktionsverminderung führen [6] und ist, bedingt durch die repetitive Schädigung, als Risikofaktor für Herzinsuffizienz belegt $[5,9,37]$. Als grundlegender und gefährlicher muss der schädigende Einfluss von überhöhten Insulinspiegeln und von Insulinresistenz (s. Abschn. „Diastolische Dysfunktion und Adipositas/Insulinresistenz") bei Typ 2 Diabetes gesehen werden $[8,16,38]$, der sich z. B. in der postprandialen Phase als Reduktion der Myokarddurchblutung um $30 \%$ auswirkt ([30]; • Abb. 6). Diese Verminderung ist speziell mit Analoginsulin im Vergleich zu anderen Insulintypen nicht nachweisbar [31]. Man kann wohl davon ausgehen, dass bei Typ 2 Diabetes angesichts des erhöhten myokardialen Sauerstoffbedarfs durch die diabetisch bedingte erhöhte Gefäßsteifigkeit ein DD auslösendes Energie-Mismatch besteht (• Abb. 4; [5]). Daten zur endothelialen Dysfunktion in der postprandialen Phase, die den Zusammenhang belegen, stehen noch aus. 


\section{Diastolische Dysfunktion und Alter}

Die physiologisch abnehmende diastolische Funktion des älteren Menschen beruht wesentlich auf vaskulären bzw. muskulären degenerativen und sklerosierenden Mechanismen und hat Einfluss auf Regulation und Effektivität der myokardialen Durchblutung. Allgemein kardiologisch sind diese physiologischen Alterungsprozesse akzeptiert, sie machen aber gerade den älteren Menschen dafür anfällig, anhand weiterer Risikofaktoren eine DD zu entwickeln.

\section{Diastolische Dysfunktion und Hypertonus}

LV Hypertrophie als Folge von arteriellem Hypertonus und dem Spätstadium hypertensive Herzerkrankung haben das Verständnis für DD als Folge des „steifen linken Ventrikels“ geprägt. Jedoch bestehen schon frühzeitig verlängerte Diffusionswege zwischen hypertrophierten Kardiozyten und Kapillaren, also eine relative Rarefizierung der Endstrombahn, und ein erhöhter myokardialer Sauerstoffbedarf wegen erhöhter Nachlast. Dieser Energie-Mismatch zwischen myokardialer Versorgung und Bedarf führt zu frühdiastolischer Dysfunktion. Zusätzlich haben viele hypertensive Patienten einen hohen Body-Mass-Index (BMI) und Insulinresistenz. Hinsichtlich Prävention besteht ein gewisses Potenzial durch die mögliche Reversibilität der DD auf metabolischer Basis (s. Abschn. „Diastolische Dysfunktion und Adipositas/Insulinresistenz").

\section{Diastolische Dysfunktion und koronare Herzkrankheit}

Stenosierende Koronarveränderungen bedingen die Reduktion der koronaren Flussreserve und Sauerstoffversorgung im abhängigen Myokard und damit eine Verminderung der diastolischen Funktionsreserve. Von endothelialer/ mikrovaskulärer Dysfunktion sind meist auch Myokardregionen von angiographisch „normalen“ Gefäßabschnitten betroffen, da Koronarkranke meist übergewichtig und häufig insulinresistent sind (s. Abschn. „Diastolische Dysfunktion und Adipositas/Insulinresistenz").

\section{Diastolische Dysfunktion und Anämie}

Anämie jeglicher Ätiologie führt zur Einschränkung der myokardialen Sauerstoffverfügbarkeit für den kardialen Energiebedarf und ist unabhängig von der Gefäßversorgung für die Beeinflussung der Funktion von Bedeutung.

\section{Diastolische Dysfunktion und chronisch obstruktive Lungenerkrankung/Schlafapnoe}

Auch ein Zusammenhang zwischen pulmonal verursachter Hypoxämie und DD ist $\mathrm{zu}$ erwarten. In diesen Formenkreis fallen insbesondere die chronisch obstruktive Lungenerkrankung und die obstruktive Schlafapnoe. Hier kommt es durch die frustrane Atemanstrengung gegen verschlossene Atemwege zu einer Zunahme von Nachlast, Wandspannung, Sympathikusaktivität und damit erhöhtem Sauerstoffverbrauch bei gleichzeitigen Sauerstoffabfällen mit Hypoxämie [36], die auch zur Modulation des kardialen autonomen Nervensystems führen können.

\section{Diastolische Dysfunktion und Niereninsuffizienz}

Beim akuten oder chronischen kardiorenalen Syndrom ist Niereninsuffizienz eng mit Herzinsuffizienz/DD assoziiert. Hier tragen die bereits erwähnten pathophysiologischen Mechanismen des renalen Hypertonus, der Hypervolämie, der Anämie sowie metabolischen Azidose $\mathrm{zu}$ einem myokardialen EnergieMismatch bei, welche auch ausgeprägte spätdiastolische Funktionsstörungen mit manifester LV Hypertrophie und Fibrose-/Kollageneinlagerung aggravieren. Auch bei leichter Niereninsuffizienz führen multiple Mediatoren wie erhöhtes Renin-Angiotensin und Sympathikotonus, chronische Entzündung und oxidativer Stress zu endothelialer Dysfunktion mit konsekutiver Auswirkung auf die frühdiastolische Funktion [23].

\section{Spätdiastolische Dysfunktion und LV Steifigkeit}

DD in der späten Diastole ist mit erhöhter LV Steifigkeit assoziiert und zeigt in Kardiozyten und Matrix Modifikationen in Abhängigkeit der multiplen Komorbiditäten. Bekanntlich führt lang andauernde LV Druckbelastung zu ausgeprägter Hypertrophie und inflammatorischen Veränderungen sowie Fibrosierung und Kollageneinlagerung [14, 17]. Auch andere Risikofaktoren zeigen infolge mikrovaskulärer endothelialer Dysfunktion bei längerem Verlauf strukturelle Modifikationen der extrazellulären Matrix [23]. Dazu gehören z. B. Lebensalter, koronare Herzkrankheit, Ischämie oder Entzündung mit Fibrosierung bzw. Kollagenmodifikationen. Spezifischer für Typ 2 Diabetes ist die Einlagerung von „advanced glycemic endproducts" (AGE) [9, 14] sowie die Einlagerung von Triglyzeriden als Steatosis [28] bei Insulinresistenz/ Adipositas.

Erhöhte kardiomyozytäre Steifigkeit wird einer Modifikation des zytoskeletalen Proteins Titin zugeschrieben, die mit oxidativem Stress und/oder der Phosphorylierung von Titin zusammenhängt. Insgesamt vermindert oxidativer Stress auch das Signaling von cGMP-PKG und die Bioverfügbarkeit von Stickstoffmonoxid, das die kalziumabhängigen Mechanismen reguliert, die zur Verbesserung der LV Relaxation und Dehnbarkeit führen [23].

Das derzeitige Verständnis der Remodeling-Prozesse hat bisher zu keiner erfolgreichen Umsetzung in kausal ansetzenden Therapiestudien geführt.

\section{Management/ Therapieoptionen}

DD hat ein breites Spektrum zugrunde liegender Risikofaktoren bzw. Komorbiditäten, die jeweils für den spezifischen Therapieansatz maßgeblich sind.

\section{Therapie/Prävention der diastolischen Dysfunktion}

Insulinresistenz wird in erster Linie durch Gewichtsabnahme bzw. durch kohlenhydratreduzierte Kost verbessert. Letztere 

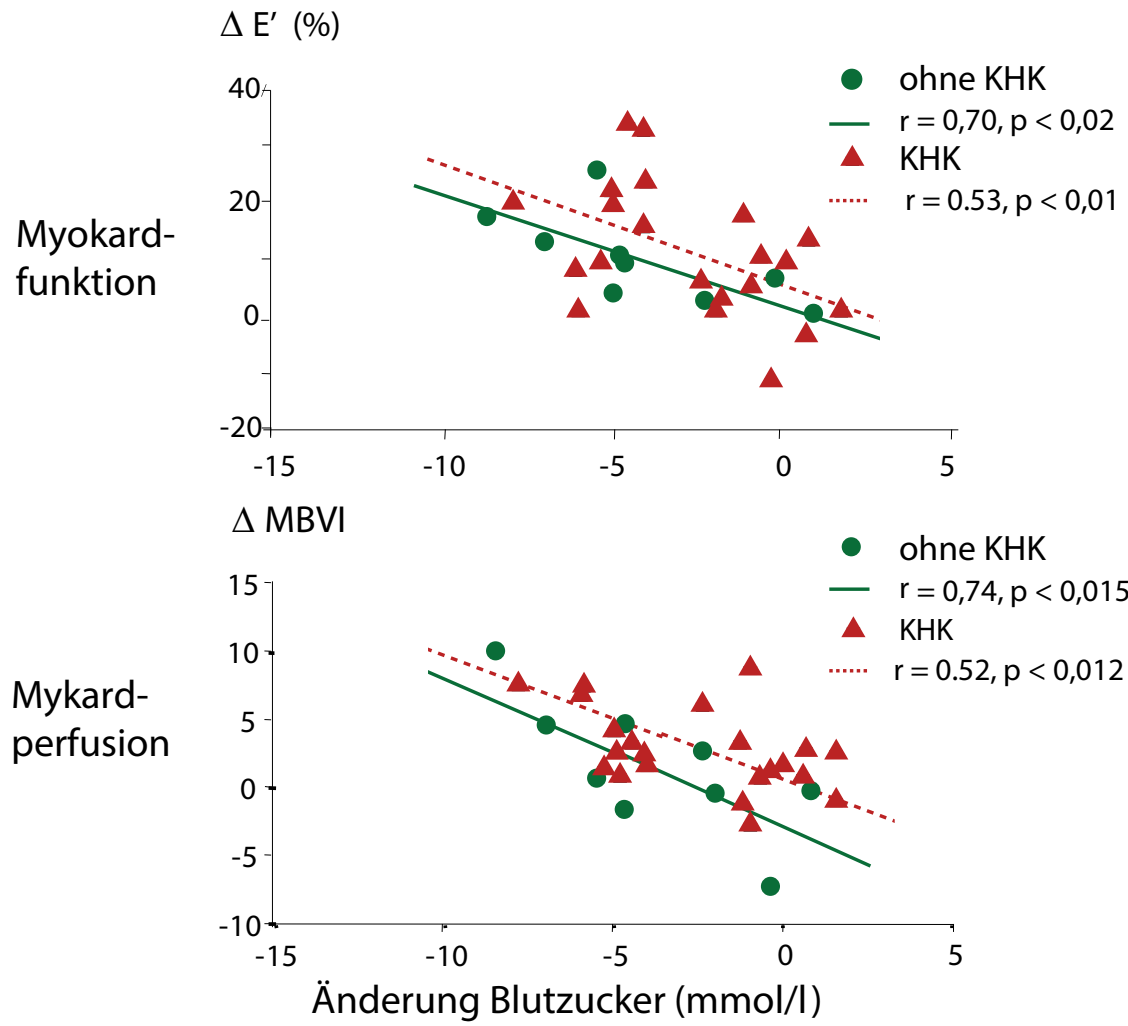

Abb. 7 A Myokardfunktion und -perfusion verbessern sich in Korrelation zur Blutzuckerreduktion durch antidiabetische Therapie bei Typ 2 Diabetes mit und ohne koronare Herzerkrankung (KHK). $\mathrm{MBVI}=$ myokardialer Blutvolumenindex. (Modifiziert nach [3])

wirkt durch die Vermeidung postprandialer Blutzucker- und Insulinspitzen sogar unabhängig von Gewichtsreduktion und erfährt dadurch mehr Akzeptanz bei Patienten. Insgesamt führt diese Kost bereits kurzfristig zu positivem Effekt für DD $[7,27]$ und, wie inzwischen in Metaanalysen beschrieben, zur Reduktion sämtlicher kardiovaskulärer Risikofaktoren [29]. Verbessernd wirkt zusätzliches Training v. a. bei gleichzeitigem Gewichtsverlust [18], ist jedoch ohne adäquate Kostmodifikation kaum effektiv.

Bei Typ 2 Diabetikern wurden die Bedeutung guter Blutzuckerkontrolle, speziell postprandial, und die Wirksamkeit von üblichen pharmakologischen Antidiabetika wie Metformin und Analoginsulinen zur Verbesserung der DD bestätigt ([2-4, 31]; - Abb. 7). Eine kausale Therapie ist gerade für Typ 2 Diabetiker die diätetische Normalisierung der Insulinresistenz über Gewichtsreduktion. Unabhängig vom Gewichtsverlust verbessert eine kohlenhydratreduzierte Kost durch Vermeidung postprandialer Blutzucker- und Insulinspitzen die DD, die
Leistungsfähigkeit und die kardiovaskulären Risikofaktoren ([7, 29]; • Abb. 8).

Bei hypertensiven Patienten gibt es zahlreiche Publikationen, nach denen leitliniengerechte antihypertensive Therapie zur Verbesserung der DD führt, so mit ACE-Hemmern, $\mathrm{AT}_{1}$-Blockern bzw. Kalziumantagonisten und für milden Hypertonus mit Diuretika bzw. $\beta$-Blockern. Zusätzliche indirekte pleiotrope Mechanismen verbessern über eine Reduktion des oxidativen Stresses die endotheliale Dysfunktion, besonders bei Patienten mit überhöhtem BMI. Es zeigten sich für letztere Patienten zur Verbesserung der DD folgende Maßnahmen als effektiv: die DASH-Diät [15], kohlenhydratreduzierte Kost [7] und zusätzliche Gabe von Statinen [23].

Die kausale Verbindung zwischen koronarer Herzerkrankung und DD zeigt sich auch in der bekannten Reversibilität der Funktionsstörung nach erfolgreicher Revaskularisation. Die konventionelle pharmakologische Therapie trägt zur mikrozirkulären myokardialen Durchblutungsverbesserung bei [11].
Für diabetische Patienten mit koronarer Herzerkrankung kann eine optimierte Zuckereinstellung zur Verbesserung von DD und Myokardperfusion führen (- Abb. 7; [3]).

Nichtkardiovaskuläre Risikofaktoren wie Anämie, chronisch obstruktive Lungenerkrankung, Schlafapnoe oder Niereninsuffizienz müssen spezifisch nach den geltenden Leitlinien therapiert werden.

\section{Prävention bzw. Therapie der HFpEF}

Es gibt derzeit noch keine Publikationen zur Prävention einer Weiterentwicklung der DD zu HFpEF. Die älteren (z. B. CHARM, I-PRESERVE) und auch die jüngsten großen pharmakologischen Studien (TOPCAT, Aldo-DHF, RELAX) haben unschlüssige Ergebnisse für die Therapieeffizienz erbracht, sodass sie den Mangel an evidenzbasierten Therapieempfehlungen für HFpEF weiterhin nicht beheben konnten [5, 23, 36]. Genannt seien noch Studien niedrigerer Evidenz, aber mit relevanten Ergebnissen für die Praxis. Es handelt es sich hier jeweils um Life-style-Modifikationen bei Adipösen, einmal um Training für überwiegend hypertensive Patienten mit BMI $31 \mathrm{~kg} / \mathrm{m}^{2}$ [12] und um die Low-Sodium-DASHDiät für hypertensives HFpEF bei BMI $35 \mathrm{~kg} / \mathrm{m}^{2}$ [15], die zu verbesserter Funktion und Symptomatik führten.

\section{Zusammenfassung und Ausblick}

Obgleich DD eine klinische Diagnose auf der Basis kardiovaskulärer und systemischer Komorbiditäten ist, hat DD wegen ihrer Prävalenz und Progression zu HFpEF hohe klinische Relevanz. Diagnostisch ist nun eine auf Gewebedoppler basierende Quantifizierung der DD möglich als Vergleich der gemessenen E' zum altersbezogenen, in einer Regressionsgleichung von gesunden Individuen errechneten Normwert E' Dieser Ansatz ist wegen der starken Altersabhängigkeit von E’ grundlegend für die korrekte Identifizierung von Risikofaktoren der DD. Zusätzlich trägt er dem dynamisch variablen Charakter der diastolischen Funktion für alle Ätiologien Rechnung und vermeidet somit 

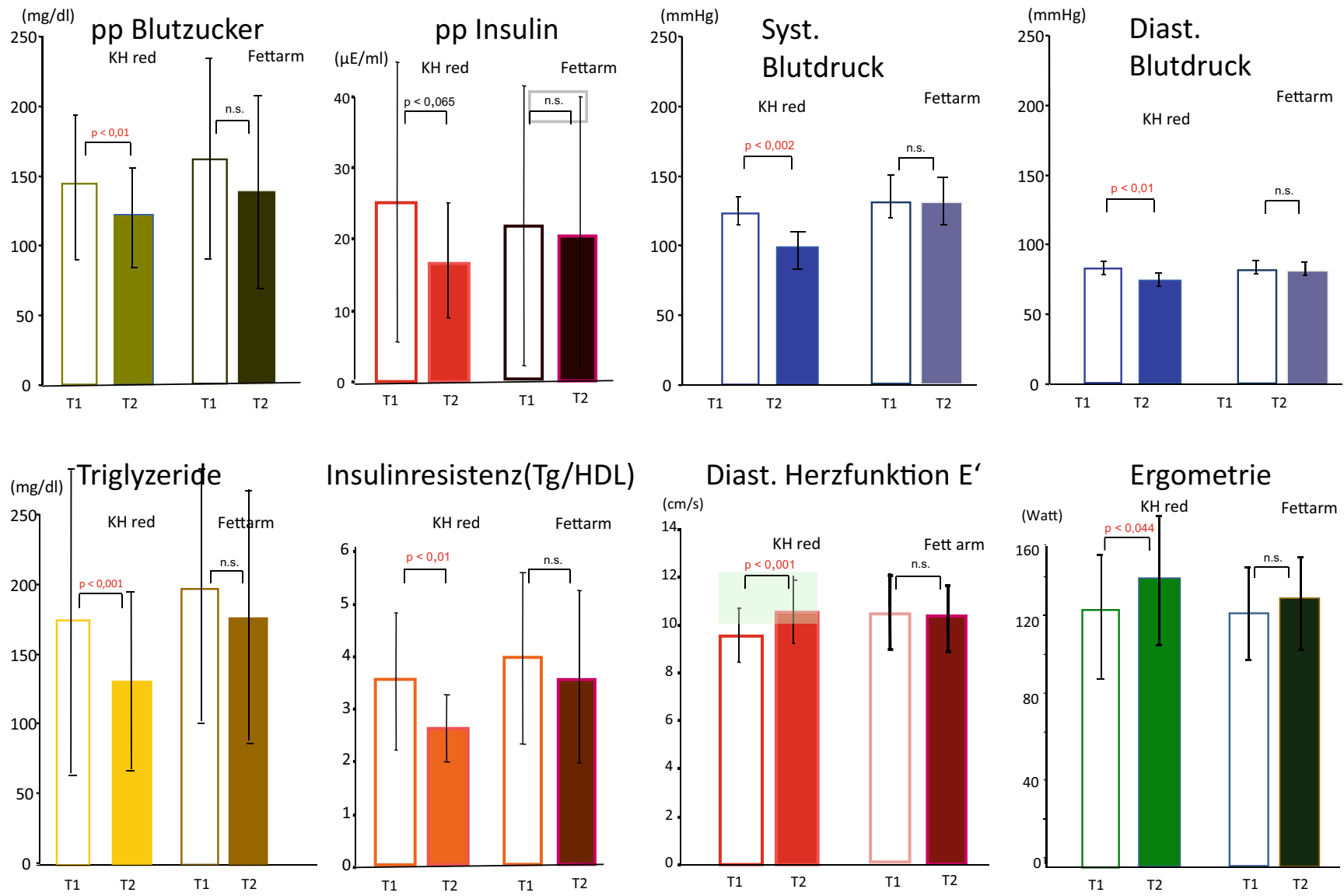

Abb. 8 A Positive Effekte kohlenhydratreduzierter Kost bei Typ 2 Diabetes auf Stoffwechsel, kardiovaskuläre Risikofaktoren und Herzfunktion im Vergleich zur traditionell empfohlenen fettarmen Kost nach 3 Wochen (T2) im Vergleich zur Ausgangsuntersuchung (T1).pp postprandial, diast. diastolisch, syst. systolisch, Tg Triglyzeride, HDL High-density-Lipoprotein. (Modifiziert nach [7])

die Probleme von E/E’ oder strukturellen LV bzw. LA Parametern für diese unterschiedlichen Krankheitsentitäten. Verbessert werden hiermit auch die Früherkennung einer Progression von DD zu HFpEF sowie die Definition geeigneter ätiologischer Einschlusskriterien für Therapiestudien. Darüber hinaus besteht erheblicher Forschungsbedarf zur endothelialen Dysfunktion als Regulans von Myokardperfusion und DD gerade auch aus metabolischer Sicht für systemische Erkrankungen und in der postprandialen Phase. Denn die reversible Natur metabolischer Abläufe zur Verbesserung von DD bietet für die betroffenen Patienten Erfolg versprechende Therapieansätze, wie hier aktuell und mit niedriger Evidenz für Insulinresistenz beschrieben werden konnte.

\section{Fazit für die Praxis}

- DD ist häufig, gerade bei Patienten mit Übergewicht/Insulinresistenz.

- Die Diagnose ist prognostisch wichtig zur Prävention von HFpEF.

- Die auf Gewebedoppler basierende Definition der DD muss wegen der physiologischen Reduktion der diastolischen Funktion um $1 \%$ pro Lebensjahr altersnormiert sein als Vergleich der individuellen $\mathrm{E}^{\prime}$ mit der errechneten altersbezogenen $E_{\text {norm }}^{\prime}$.

- Die Vielfalt ätiologischer Faktoren von DD verlangt eine genaue Diagnostik und spezifische Therapie.

- Insulinresistenz und endotheliale Dysfunktion haben hierbei das Potenzial guter Reversibilität.

- Die Therapieoptionen zur Normalisierung von Insulinresistenz (Kost und Training) sollten ausgeschöpft werden.

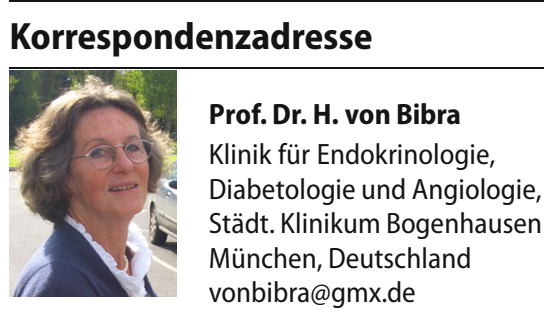

\section{Einhaltung ethischer Richtlinien}

Interessenkonflikt. H. von Bibra und W. Paulus geben an, dass kein Interessenkonflikt besteht.

Dieser Beitrag beinhaltet keine Studien an Menschen oder Tieren.

Open Access. This article is distributed under the terms of the Creative Commons Attribution 4.0 International License (http://creativecommons.org/ licenses/by/4.0/), which permits unrestricted use, distribution, and reproduction in any medium, provided you give appropriate credit to the original author(s) and the source, provide a link to the Creative Commons license, and indicate if changes were made. 


\section{Literatur}

1. Abhyaratna WP, Marwick TH, Smith WT et al (2006) Characteristics of left ventricular diastolic dysfunction and risk of heart failure. Heart 92:1259-1264

2. Andersson C, Sogaard P, Hoffmann S et al (2010) Metformin is associated with improved left ventricular diastolic function measured by tissue Doppler imaging in patients with diabetes. Eur J Endocrinol 163:593-599

3. von Bibra H, Hansen A, Dounis V et al (2004) Augmented metabolic control improves myocardial diastolic function and perfusion in patients with type 2 diabetes mellitus. Heart 90:1483-1484

4. von Bibra H, St John Sutton M (2010) Diastolic dysfunction in diabetes and the metabolic sndrome - promising potential for diagnosis and prognosis. Diabetologia 53:1033-1045

5. von Bibra H, St John Sutton M (2011) Impact of diabetes on postinfarction heart failure and left ventricular remodeling. Curr Heart Fail Rep 8:242-251

6. von Bibra H, St John Sutton M, Schuster T et al (2013) Oxidative stress after a carbohydrate meal contributes to the deterioration of diastolic cardiac function in non-hypertensive insulintreated patients with moderately well controlled type 2 diabetes. Horm Metab Res 45:449-455

7. von Bibra H, Wulf G, St John Sutton M et al (2014) A low-carbohydrate/high-protein diet improves diastolic cardiac function and the metabolic syndrome in overweight-obese patients with type 2 diabetes. IJC Metab Endocr 2:11-18

8. von Bibra H, Paulus WJ, St John Sutton M et al (2015) Quantification of diastolic dysfunction via the age dependence of diastolic function - impact of insulin resistance with and without type 2 diabetes. Int J Cardiol 182:368-374

9. Boudina S, Dale AE (2007) Diabetic cardiomyopathy revisited. Circulation 115:3213-3223

10. deKnegt MC, Bierung-Sorensen $T$, Sogaard $P$ et al (2014) Concordance and reproducibility between $\mathrm{M}$-mode, tissue Doppler imaging, and two dimensional strain imaging in the assessment of mitral annular displacement and velocity in patients with various heart conditions. Eur Heart J Cardiovasc Imaging 15:62-69

11. Dounis V, Siegmund T, Jensen J et al (2006) Global myocardial perfusion and diastolic function are impaired to a similar extent in patients with type 2 diabetes mellitus and in patients with coronary artery disease - evaluation by contrast echocardiography and pulsed tissue Doppler. Diabetologia 49:2729-2740

12. Edelmann F, Gelbrich G, Düngen HD et al (2011) Exercise training improves exercise capacity and diastolic function in patients with heart failure with preserved ejection fraction. J Am Coll Cardio 58:1781-1792

13. Emery WT, Jadavji I, Choy JB et al (2008) Investigating the European Society of Cardiology Diastology Guidelines in a practical scenario. Eur J Echocardiogr 9:685-691

14. van Heerebeek L, Hamdani N, Handoko L et al (2008) Diastolic stiffness of the failing heart: importance of fibrosis, advanced glycation endproducts and myocyte resting tension. Circulation 117:43-51

15. Hummel SL, Seymour EM, Brook RD et al (2013) Low-sodium DASH dietimproves diastolicfunction and ventricular-arterial coupling in hypertensive heart failure with preserved ejection fraction. Circ Heart Fail 6:1165-1171
16. Hwang YC, Jee JH, Kang M et al (2012) Metabolic syndrome and insulin resistance are associated with abnormal left ventricular diastolic function and structure independent of blood pressure and fasting plasma glucose level. Int J Cardiol 179:107-111

17. Kasner M, Westermann D, Lopez B et al (2011) Diastolic tissue Doppler indexes correlate with the degree of collagen expression and crosslinking in heart failure and normal ejection fraction. J Am Coll Cardiol 57:977-985

18. Kosmala W, O'Moore-Sullivan T, Plaksej R et al (2009) Improvement of left ventricular function by lifestyle intervention in obesity: contributions of weight loss and reduced insulin resistance. Diabetologia 52:2306-2316

19. Lam CS (2010) HFpEF: Invasive solution to diagnostic confusion? J Am Coll Cardiol 55:1711-1712

20. Lang RM, Bierig M, Devereux R et al (2006) American Society of Echocardiography's Nomenclature and Standards Committee; Task Force on Chamber Quantification; American College of Cardiology Echocardiographic Committee, American Heart Association, European Association of Echocardiography, European Society of Cardiology recommendations for chamber quantification. Eur JEchocardiogr 7:79-108

21. Mogelvang R, Sogaard P, Pedersen SA et al (2009) Cardiac dysfunction assessed by echocardiographic tissue Doppler imaging is an independent predictor of mortality in the general population. Circulation 119:2679-2685

22. Paulus WJ, Tschöpe C, Sandermann JE et al (2007) How to diagnose diastolic heart failure: a consensus statement on the diagnosis of heart failure with normal left ventricular ejection fraction by the HeartFailure and Echocardiography Associations of the European Society of Cardiology Eur Heart J 28:2539-2550

23. Paulus W, Tschöpe C (2013) A novel paradigm for heart failure with preserved ejection fraction: comorbidities drive myocardial dysfunction and remodeling through coronary microvascular endothelial inflammation. J Am Coll Cardiol 62:263-271

24. Penicka M, Bartunek J, Trakalova H (2010) Heart failure with preserved ejection fraction in outpatients with unexplained dyspnea: a pressure-volume loop analysis. J Am Coll Cardiol 55:1701-1710

25. Phan TT, Abozguia K, Shivu G et al (2009) Heart failure with preserved ejection fraction is characterized by dynamic impairment of active relaxation and contraction of the left ventricle on exercise and associated with myocardial energy deficiency. J Am Coll Cardiol 54:4002-4009

26. Redfield MM, Jacobsen SJ, Burnett JC Jr et al (2003) Burden of systolic and diastolic ventricular dysfunction in the community: appreciating the scope of the heart failure epidemic. JAMA 296:194-202

27. Rider OJ, Francis JM, Ali MK et al (2009) Beneficial cardiovascular effects of bariatric surgical and dietary weight loss in obesity. J Am Coll Cardiol 54:718-726

28. Rijzewjik LJ, van der Meer RW, Smit JW et al (2008) Myocardial steatosis is an independent predictor of diastolic dysfunction in type 2 diabetes mellitus. J Am Coll Cardiol 52:1793-1799

29. Santos FL, Esteves SS, da Costa PA et al (2012) Systematic review and meta-analysis of clinical trials of the effects of low carbohydrate diets on cardiovascular riskfactors. Obes Rev 13:1048-1066
30. Scognamiglio R, Negut C, De Kreutzenberg SV et al (2005) Postprandial myocardial perfusion in healthy subjects and in type 2 diabetic patients. Circulation 112:179-184

31. Scognamiglio R, Negut $C$, de Kreutzenberg $S$ et al (2006) Effects of different insulin regimens on postprandial myocardial perfusion defects in type 2 diabetic patients. Diabetes Care 29:95-100

32. Serizawa T, Vogel WM, Apstein CS et al (1980) Comparison of acute alterations in left ventricular relaxation and diastolic chamber stiffness induced by hypoxia and ischemia. JClin Invest 68:91-102

33. Stritzke J, Markus MR, Duderstadt S et al (2009) The aging process of the heart: obesity is the main risk factor for left atrial enlargement during aging. J Am Coll Cardiol 54:1982-1989

34. Tsang TS, Abhayratna WP, Barnes ME et al (2006) Prediction of cardiovascular outcomes with left atrial size - is volume superior to area or diameter? JAm Coll Cardiol 47:1018-1023

35. Tschöpe C, Paulus WJ (2009) Is echocardiographic evaluation of diastolic function useful in determining clinical care? - Doppler echocardiography yields dubious estimates of left ventricular diastolic pressure. Circulation 120:810-820

36. Wachter R, Luthje L, Klemmstein D et al (2013) Impact of obstructive sleep apnea on diastolic function. Eur Respir J41:3776-3383

37. Wan SH, Vogel MW, Chen HH (2014) Pre-clinical diastolic dysfunction. J Am Coll Cardiol 63:407-416

38. Witteles RM, Fowler MB (2008) Insulin-resistant cardiomyopathy - clinical evidence, mechanisms, and treatmentoptions.JAm Coll Cardiol 51:93-102

39. Wong C, Marwick TH (2007) Obesity cardiomyopathy: pathogensis and pathophysiology. Nat Clin Pract Cardiovasc Med 4:436-443

40. Yu CM, Sanderson JE, Marwick TH et al (2007) Tissue Doppler Imaging. A new prognosticator for cardiovascular disease. J Am Coll Cardiol 49:1903-1914 